\title{
Statistical approximation by positive linear operators
}

\author{
by \\ O. Duman and C. Orhan (Ankara)
}

\begin{abstract}
Using $A$-statistical convergence, we prove a Korovkin type approximation theorem which concerns the problem of approximating a function $f$ by means of a sequence $\left\{T_{n}(f ; x)\right\}$ of positive linear operators acting from a weighted space $C_{\varrho_{1}}$ into a weighted space $B_{\varrho_{2}}$.
\end{abstract}

1. Introduction. The sequences of some classical approximation operators tend to converge to the values of the function they approximate. However, at points of discontinuity, they often converge to the average of the left and right limits of the function. There are, however, some exceptions, such as the interpolation operators of Hermite-Fejér [2] that do not converge at points of simple discontinuity. In this case, the matrix summability methods of Cesàro type are applicable to correct the lack of convergence [3]. Statistical convergence, which is a regular non-matrix summability method, is also effective in "summing" divergent sequences [7], [9], [10]. Recently, its use in approximation theory has been considered in [6], [13]. The aim of this paper is to use $A$-statistical convergence to study Korovkin type approximation of a function $f$ by means of a sequence $\left\{T_{n}(f ; x)\right\}$ of positive linear operators from a weighted space $C_{\varrho_{1}}$ into a weighted space $B_{\varrho_{2}}$.

Approximation theory has important applications in various areas of functional analysis, and in numerical solution of differential and integral equations [1], [5], [18].

Before proceeding we recall some notation on statistical convergence. Let $A=\left(a_{j n}\right)$ be an infinite summability matrix. For a given sequence $x:=\left(x_{n}\right)$, the $A$-transform of $x$, denoted by $A x:=\left((A x)_{j}\right)$, is given by $(A x)_{j}=\sum_{n=1}^{\infty} a_{j n} x_{n}$, provided the series converges for each $j$. We say that $A$ is regular if $\lim _{j}(A x)_{j}=L$ whenever $\lim _{j} x_{j}=L$ (see [14]). Assume now that $A$ is a non-negative regular summability matrix and $K$ is a subset of $\mathbb{N}$, the set of all natural numbers. The A-density of $K$ is defined by

2000 Mathematics Subject Classification: 41A25, 41A36, 47B38.

Key words and phrases: $A$-density, $A$-statistical convergence, sequence of positive linear operators, weight function, weighted space, Korovkin type theorem. 
$\delta_{A}(K):=\lim _{j} \sum_{n=1}^{\infty} a_{j n} \chi_{K}(n)$ provided the limit exists, where $\chi_{K}$ is the characteristic function of $K$. A sequence $x:=\left(x_{n}\right)$ is said to be $A$-statistically convergent to a number $L$ if, for every $\varepsilon>0, \delta_{A}\left\{n \in \mathbb{N}:\left|x_{n}-L\right| \geq \varepsilon\right\}=0$; or equivalently

$$
\lim _{j} \sum_{n:\left|x_{n}-L\right| \geq \varepsilon} a_{j n}=0 .
$$

We denote this limit by $\operatorname{st}_{A}$-lim $x=L$ ([4], [8], [17], [19]). For $A=C_{1}$, the Cesàro matrix, $A$-statistical convergence reduces to statistical convergence ([7], [9], [10]). We note that if $A=\left(a_{j n}\right)$ is a non-negative regular summability matrix for which $\lim _{j} \max _{n}\left\{a_{j n}\right\}=0$, then $A$-statistical convergence is stronger than convergence [17].

It should be noted that the concept of $A$-statistical convergence may also be given in normed spaces: Assume $(X,\|\cdot\|)$ is a normed space and $u=\left(u_{n}\right)$ is an $X$-valued sequence. Then $\left(u_{n}\right)$ is said to be A-statistically convergent to $u_{0} \in X$ if, for every $\varepsilon>0, \delta_{A}\left\{n \in \mathbb{N}:\left\|u_{n}-u_{0}\right\| \geq \varepsilon\right\}=0$ (see [15], [16]). We recall that $x=\left(x_{n}\right)$ is $A$-statistically convergent to $L$ if and only if there exists a subsequence $\left\{x_{n(k)}\right\}$ of $x$ such that $\delta_{A}\{n(k): k \in \mathbb{N}\}=1$ and $\lim _{k} x_{n(k)}=L$ (see [17], [19]). The same result also holds in normed spaces $([15],[16])$.

Now we recall the concepts of weight functions and weighted spaces considered in [11], [12]. Let $\mathbb{R}$ denote the set of real numbers. A real-valued function $\varrho$ is called a weight function if it is continuous on $\mathbb{R}$ and

$$
\lim _{|x| \rightarrow \infty} \varrho(x)=\infty, \quad \varrho(x) \geq 1 \quad(\text { for all } x \in \mathbb{R}) .
$$

The space of real-valued functions $f$ defined on $\mathbb{R}$ and satisfying $|f(x)| \leq$ $M_{f} \varrho(x)$ (for all $x \in \mathbb{R}$ ) is called the weighted space and denoted by $B_{\varrho}$, where $M_{f}$ is a constant depending on the function $f$. The weighted subspace $C_{\varrho}$ of $B_{\varrho}$ is given by

$$
C_{\varrho}:=\left\{f \in B_{\varrho}: f \text { is continuous on } \mathbb{R}\right\} .
$$

The spaces $B_{\varrho}$ and $C_{\varrho}$ are Banach spaces with the norm

$$
\|f\|_{\varrho}:=\sup _{x \in \mathbb{R}} \frac{|f(x)|}{\varrho(x)} .
$$

Now let $\varrho_{1}$ and $\varrho_{2}$ be two weight functions satisfying (1). Assume also that

$$
\lim _{|x| \rightarrow \infty} \frac{\varrho_{1}(x)}{\varrho_{2}(x)}=0 .
$$

If $T$ is a positive linear operator from $C_{\varrho_{1}}$ into $B_{\varrho_{2}}$, then the operator norm $\|T\|_{C_{\varrho_{1}} \rightarrow B_{\varrho_{2}}}$ is given by

$$
\|T\|_{C_{\varrho_{1}} \rightarrow B_{\varrho_{2}}}:=\sup _{\|f\|_{\varrho_{1}}=1}\|T f\|_{\varrho_{2}} .
$$


The following approximation theorem for a sequence of positive linear operators acting from $C_{\varrho_{1}}$ into $B_{\varrho_{2}}$ may be found in [11] and [12].

TheOREM A. Assume that $\varrho_{1}$ and $\varrho_{2}$ are weight functions satisfying (2) and $\left\{L_{n}\right\}$ is a sequence of positive linear operators from $C_{\varrho_{1}}$ into $B_{\varrho_{2}}$. Then $\lim _{n}\left\|L_{n} f-f\right\|_{\varrho_{2}}=0$ for all $f \in C_{\varrho_{1}}$ if and only if $\lim _{n}\left\|L_{n} F_{v}-F_{v}\right\|_{\varrho_{1}}=0$ for $v=0,1,2$, where

$$
F_{v}(x)=\frac{x^{v} \varrho_{1}(x)}{1+x^{2}}, \quad v=0,1,2 .
$$

In the present paper, we give an analog of Theorem A with the ordinary limit operator replaced by an $A$-statistical limit operator. We will also exhibit an example of a sequence of positive linear operators to which Theorem A does not apply but our $A$-statistical approximation theorem does.

2. Statistical approximation in weighted spaces. In this section we will obtain a Korovkin type approximation theorem for $A$-statistical convergence of a sequence of positive linear operators acting from $C_{\varrho_{1}}$ into $B_{\varrho_{2}}$.

We require the following lemmas.

Lemma 1. Let $A=\left(a_{j n}\right)$ be a non-negative regular summability matrix and let $\left\{T_{n}\right\}$ be a sequence of positive linear operators from $C_{\varrho_{1}}$ into $B_{\varrho_{2}}$, where $\varrho_{1}$ and $\varrho_{2}$ satisfy condition (2). Assume that there exists a number $M>0$ such that

$$
\delta_{A}\left\{n \in \mathbb{N}:\left\|T_{n}\right\|_{C_{\varrho_{1}} \rightarrow B_{\varrho_{1}}} \leq M\right\}=1 .
$$

If

$$
\mathrm{st}_{A^{-}} \lim _{n} \sup _{\|f\|_{\varrho_{1}}=1} \sup _{|x| \leq s} \frac{\left|T_{n}(f ; x)\right|}{\varrho_{1}(x)}=0 \quad \text { for any } s \in \mathbb{R}
$$

then

$$
\mathrm{st}_{A^{-}} \lim _{n}\left\|T_{n}\right\|_{C_{\varrho_{1}} \rightarrow B_{\varrho_{2}}}=0 .
$$

Proof. By (2), given $\varepsilon>0$, there exists a number $s_{0}$ such that $\varrho_{1}(x) \leq$ $(\varepsilon / M) \varrho_{2}(x)$ for $|x|>s_{0}$. Also, by the continuity of $\varrho_{1} / \varrho_{2}$, there exists $C>0$ such that $\varrho_{1}(x) \leq C \varrho_{2}(x)$ whenever $|x| \leq s_{0}$. Let

$$
K:=\left\{n \in \mathbb{N}:\left\|T_{n}\right\|_{C_{\varrho_{1}} \rightarrow B_{\varrho_{1}}} \leq M\right\} .
$$

By (3), $\delta_{A}(K)=1$. Then, for all $n \in K$, by (5) we have

$$
\begin{aligned}
\left\|T_{n}\right\|_{C_{\varrho_{1}} \rightarrow B_{\varrho_{2}}} & =\sup _{\|f\|_{\varrho_{1}}=1} \sup _{x \in \mathbb{R}} \frac{\left|T_{n}(f ; x)\right|}{\varrho_{2}(x)} \\
& \leq \sup _{\|f\|_{\varrho_{1}}=1} \sup _{|x| \leq s_{0}} \frac{\left|T_{n}(f ; x)\right|}{\varrho_{2}(x)}+\sup _{\|f\|_{\varrho_{1}}=1} \sup _{|x|>s_{0}} \frac{\left|T_{n}(f ; x)\right|}{\varrho_{2}(x)}
\end{aligned}
$$




$$
\begin{aligned}
& \leq C \sup _{\|f\|_{\varrho_{1}}=1} \sup _{|x| \leq s_{0}} \frac{\left|T_{n}(f ; x)\right|}{\varrho_{1}(x)}+\frac{\varepsilon}{M} \sup _{\|f\|_{\varrho_{1}}=1} \sup _{x \in \mathbb{R}} \frac{\left|T_{n}(f ; x)\right|}{\varrho_{1}(x)} \\
& \leq C \varphi_{n}\left(s_{0}\right)+\frac{\varepsilon}{M}\left\|T_{n}\right\|_{C_{\varrho_{1}} \rightarrow B_{\varrho_{1}}} \leq C \varphi_{n}\left(s_{0}\right)+\varepsilon
\end{aligned}
$$

where

$$
\varphi_{n}\left(s_{0}\right):=\sup _{\|f\|_{\varrho_{1}}=1} \sup _{|x| \leq s_{0}} \frac{\left|T_{n}(f ; x)\right|}{\varrho_{1}(x)} .
$$

Now for a given $r>0$ choose $\varepsilon>0$ such that $\varepsilon<r$. Thus

$$
\sum_{n \in K:\left\|T_{n}\right\|_{C_{\varrho_{1}} \rightarrow \varrho_{\varrho_{2}} \geq r}} a_{j n} \leq \sum_{n \in K: C \varphi_{n}\left(s_{0}\right) \geq r-\varepsilon} a_{j n} .
$$

Hence, letting $j \rightarrow \infty$ in (6) and taking (4) into account, we get the result.

Lemma 2. Let $A=\left(a_{j n}\right), \varrho_{1}$ and $\varrho_{2}$ be as in Lemma 1. Let $\left\{T_{n}\right\}$ be a sequence of positive linear operators from $C_{\varrho_{1}}$ into $B_{\varrho_{2}}$ for which (3) holds for some $M>0$. If , for any $s \in \mathbb{R}$,

$$
\mathrm{st}_{A}-\lim _{n} \sup _{\|f\|_{\varrho_{1}}=1} \sup _{|x| \leq s}\left|T_{n}(f ; x)-f(x)\right|=0,
$$

then

$$
\operatorname{st}_{A}-\lim _{n}\left\|T_{n} f-f\right\|_{\varrho_{2}}=0 \quad \text { for all } f \in C_{\varrho_{1}} .
$$

Proof. Let $E$ be the identity operator on $C_{\varrho_{1}}$ and let $L_{n}:=T_{n}-E, U:=$ $\left\{n \in \mathbb{N}:\left\|T_{n}\right\|_{C_{\varrho_{1}} \rightarrow B_{\varrho_{1}}} \leq M\right\}$ and $V:=\left\{n \in \mathbb{N}:\left\|L_{n}\right\|_{C_{\varrho_{1}} \rightarrow B_{\varrho_{1}}} \leq M+1\right\}$. Since $\left\|L_{n}\right\|_{C_{\varrho_{1}} \rightarrow B_{\varrho_{1}}} \leq\left\|T_{n}\right\|_{C_{\varrho_{1}} \rightarrow B_{\varrho_{1}}}+1$, we have $U \subseteq V$. Since $\delta_{A}(U)=1$, we have $\delta_{A}(V)=1$. As $\varrho_{1} \geq 1$ on $\mathbb{R}$, we get, for any $s \in \mathbb{R}$,

$$
\begin{aligned}
\sup _{\|f\|_{\varrho_{1}=1} \sup _{|x| \leq s} \frac{\left|L_{n}(f ; x)\right|}{\varrho_{1}(x)}} & \leq \sup _{\|f\|_{\varrho_{1}}=1} \sup _{|x| \leq s}\left|L_{n}(f ; x)\right| \\
& =\sup _{\|f\|_{\varrho_{1}}=1} \sup _{|x| \leq s}\left|T_{n}(f ; x)-f(x)\right| .
\end{aligned}
$$

From (7) it follows that

$$
\operatorname{st}_{A^{-}} \lim _{n} \sup _{\|f\|_{\varrho_{1}}=1} \sup _{|x| \leq s} \frac{\left|L_{n}(f ; x)\right|}{\varrho_{1}(x)}=0 .
$$

Hence the sequence $\left\{L_{n}\right\}$ satisfies all the conditions of Lemma 1. So we have

$$
\operatorname{st}_{A^{-}} \lim _{n}\left\|L_{n}\right\|_{C_{\varrho_{1}} \rightarrow B_{\varrho_{2}}}=0 \text {. }
$$

Combining this with the fact that

$$
\left.\left\|L_{n} f\right\|_{\varrho_{2}} \leq\left\|L_{n}\right\|_{C_{\varrho_{1}} \rightarrow B_{\varrho_{2}}}\|f\|_{\varrho_{1}} \quad \text { (for all } f \in C_{\varrho_{1}}\right),
$$

we immediately conclude that

$$
\operatorname{st}_{A^{-}} \lim _{n}\left\|L_{n} f\right\|_{\varrho_{2}}=\operatorname{st}_{A^{-}} \lim _{n}\left\|T_{n} f-f\right\|_{\varrho_{2}}=0 \text {. }
$$


Now we present the following main result.

Theorem 3. Let $A=\left(a_{j n}\right), \varrho_{1}$ and $\varrho_{2}$ be as in Lemma 1. Assume that $\left\{T_{n}\right\}$ is a sequence of positive linear operators acting from $C_{\varrho_{1}}$ into $B_{\varrho_{2}}$. Then

$$
\text { st }_{A}-\lim _{n}\left\|T_{n} f-f\right\|_{\varrho_{2}}=0 \quad \text { for all } f \in C_{\varrho_{1}}
$$

if and only if

$$
\mathrm{st}_{A}-\lim _{n}\left\|T_{n} F_{v}-F_{v}\right\|_{\varrho_{1}}=0 \quad(v=0,1,2),
$$

where

$$
F_{v}(x)=\frac{x^{v} \varrho_{1}(x)}{1+x^{2}} \quad(v=0,1,2) .
$$

Proof. Since each $F_{v}$ belongs to $C_{\varrho_{1}}$, it is clear that (8) implies (9). Conversely, assume that (9) holds true. We first prove that (3) holds for some $M>0$.

By (9), for each $v=0,1,2$, there exists a set $K_{v} \subseteq \mathbb{N}$ such that $\delta_{A}\left(K_{v}\right)=1$ and $\lim _{n \in K_{v}}\left\|T_{n} F_{v}-F_{v}\right\|_{\varrho_{1}}=0$, i.e., given $\varepsilon>0$ there exists $N_{v}(\varepsilon)$ such that for all $n \in K_{v}$ and $n \geq N_{v}(\varepsilon)$ we have $\left\|T_{n} F_{v}-F_{v}\right\|_{\varrho_{1}}<\varepsilon$. Hence there is a positive number $M_{v}$ such that $\left\|T_{n} F_{v}-F_{v}\right\|_{\varrho_{1}} \leq M_{v}$ for every $n \in K_{v}$. Let $K:=K_{0} \cap K_{1} \cap K_{2}$. Observe that $\delta_{A}(K)=1$. So, for every $n \in K$, we have

$$
\begin{aligned}
\left\|T_{n}\right\|_{C_{\varrho_{1}} \rightarrow B_{\varrho_{1}}} & =\left\|T_{n} \varrho_{1}\right\|_{\varrho_{1}} \leq\left\|T_{n} \varrho_{1}-\varrho_{1}\right\|_{\varrho_{1}}+1 \\
& \leq\left\|T_{n} F_{2}-F_{2}\right\|_{\varrho_{1}}+\left\|T_{n} F_{0}-F_{0}\right\|_{\varrho_{1}}+1 \leq M,
\end{aligned}
$$

where $M:=1+M_{0}+M_{2}$. This implies that $K \subseteq\left\{n:\left\|T_{n}\right\|_{C_{\varrho_{1}} \rightarrow B_{\varrho_{1}}} \leq M\right\}$, which yields (3).

We now prove that condition (7) holds. To see this we write

$$
\begin{aligned}
T_{n}\left((t-x)^{2} F_{0}(t) ; x\right)= & T_{n}\left(t^{2} F_{0}(t) ; x\right)-2 x T_{n}\left(t F_{0}(t) ; x\right)+x^{2} T_{n}\left(F_{0}(t) ; x\right) \\
\leq & \left|T_{n}\left(F_{2}(t) ; x\right)-F_{2}(x)\right|+2|x|\left|T_{n}\left(F_{1}(t) ; x\right)-F_{1}(x)\right| \\
& +x^{2}\left|T_{n}\left(F_{0}(t) ; x\right)-F_{0}(x)\right| .
\end{aligned}
$$

Hence for any $s \in \mathbb{R}$ and $n \in K$ we get

$$
\begin{aligned}
u_{n} & :=\sup _{|x| \leq s} T_{n}\left((t-x)^{2} F_{0}(t) ; x\right) \\
& \leq B\left\{\left\|T_{n} F_{2}-F_{2}\right\|_{\varrho_{1}}+\left\|T_{n} F_{1}-F_{1}\right\|_{\varrho_{1}}+\left\|T_{n} F_{0}-F_{0}\right\|_{\varrho_{1}}\right\},
\end{aligned}
$$

where $B:=\max \left\{1,2 \sup _{|x| \leq s}|x| \varrho_{1}(x), \max _{|x| \leq s} x^{2} \varrho_{1}(x)\right\}$.

Now let $f \in C_{\varrho_{1}}$ and let $|x| \leq s$. Since $f$ is continuous on $\mathbb{R}$, given $\varepsilon>0$, there exists a $\delta>0$ such that $|f(t)-f(x)|<\varepsilon$ for all $t, x$ with $|t-x|<\delta$. When $|t-x| \geq \delta$, we have

$$
\begin{aligned}
|f(t)-f(x)| & \leq 2 M_{f} \varrho_{1}(x) \varrho_{1}(t)=2 M_{f} \varrho_{1}(x) F_{0}(t)\left(1+t^{2}\right) \\
& \leq 4 M_{f} \varrho_{1}(x) F_{0}(t)\left(1+x^{2}+(t-x)^{2}\right)
\end{aligned}
$$




$$
\begin{aligned}
& =4 M_{f} \varrho_{1}(x) F_{0}(t)(t-x)^{2}\left(\frac{1+x^{2}}{(t-x)^{2}}+1\right) \\
& \leq K_{\varrho_{1}}(x)(t-x)^{2} F_{0}(t),
\end{aligned}
$$

where $K_{\varrho_{1}}(x):=4 M_{f} \varrho_{1}(x)\left(\left(1+x^{2}\right) / \delta^{2}+1\right)$. So, for all $t \in \mathbb{R}$ and $|x| \leq s$, we see that

$$
|f(t)-f(x)|<\varepsilon+K_{\varrho_{1}}(x)(t-x)^{2} F_{0}(t) .
$$

It follows from (11) that

$$
\begin{aligned}
\left|T_{n}(f(t) ; x)-f(x)\right| \leq & T_{n}(|f(t)-f(x)| ; x)+|f(x)|\left|T_{n}(1 ; x)-1\right| \\
< & \varepsilon T_{n}(1, x)+K_{\varrho_{1}}(x) T_{n}\left((t-x)^{2} F_{0}(t) ; x\right) \\
& +|f(x)|\left|T_{n}(1 ; x)-1\right| .
\end{aligned}
$$

This also implies, for any $s \in \mathbb{R}$, that

$$
\begin{aligned}
v_{n}:= & \sup _{\|f\|_{\varrho_{1}}=1} \sup _{|x| \leq s}\left|T_{n}(f(t) ; x)-f(x)\right| \\
< & C_{1} \varepsilon\left\|T_{n}(1, x)\right\|_{\varrho_{1}}+C_{2} \sup _{|x| \leq s} T_{n}\left((t-x)^{2} F_{0}(t) ; x\right) \\
& +C_{3} \sup _{|x| \leq s}\left|T_{n}(1 ; x)-1\right|,
\end{aligned}
$$

where $C_{1}:=\sup _{|x| \leq s} \varrho_{1}(x), C_{2}:=\sup _{|x| \leq s} K_{\varrho_{1}}(x)$ and $C_{3}:=\sup _{|x| \leq s}|f(x)|$.

Since $\left\|T_{n}(1, x)\right\|_{\varrho_{1}} \leq\left\|T_{n}\left(\varrho_{1}, x\right)\right\|_{\varrho_{1}}=\left\|T_{n}\right\|_{C_{\varrho_{1}} \rightarrow B_{\varrho_{1}}}$, it follows from (12), for all $n \in K$, that

$$
v_{n} \leq M C_{1} \varepsilon+C_{2} u_{n}+C_{3} \sup _{|x| \leq s}\left|T_{n}(1 ; x)-1\right| .
$$

Since $F_{0} \in C_{\varrho_{1}}$ and

$$
F_{0}(x)\left|T_{n}(1 ; x)-1\right| \leq\left|T_{n}\left(F_{0}(t) ; x\right)-F_{0}(x)\right|+\left|T_{n}\left(F_{0}(t)-F_{0}(x) ; x\right)\right|,
$$

we have, by (11),

$$
\begin{aligned}
\left|T_{n}(1 ; x)-1\right|< & \frac{1}{F_{0}(x)}\left\{\left|T_{n}\left(F_{0}(t) ; x\right)-F_{0}(x)\right|+\varepsilon T_{n}(1 ; x)\right. \\
& \left.+K_{\varrho_{1}}(x) T_{n}\left((t-x)^{2} F_{0}(t) ; x\right)\right\} .
\end{aligned}
$$

So we conclude, for any $s \in \mathbb{R}$ and all $n \in K$, that

$$
\sup _{|x| \leq s}\left|T_{n}(1 ; x)-1\right| \leq C_{4}\left\{\left\|T_{n} F_{0}-F_{0}\right\|_{\varrho_{1}}+\varepsilon M+C_{2} u_{n}\right\},
$$

where $C_{4}:=\sup _{|x| \leq s} \varrho_{1}(x) / F_{0}(x)$. Taking (10), (13) and (14) into account, for all $n \in K$, we obtain

$$
v_{n} \leq C \varepsilon+C\left\{\left\|T_{n} F_{0}-F_{0}\right\|_{\varrho_{1}}+\left\|T_{n} F_{1}-F_{1}\right\|_{\varrho_{1}}+\left\|T_{n} F_{2}-F_{2}\right\|_{\varrho_{1}}\right\},
$$

where $C:=\max \left\{M\left(C_{1}+C_{3} C_{4}\right), B C_{2}+C_{3} C_{4}+B C_{2} C_{3} C_{4}\right\}$. 
Now for a given $r>0$, choose $\varepsilon>0$ such that $C \varepsilon<r$. Define

$$
\begin{aligned}
D:=\left\{n \in K:\left\|T_{n} F_{0}-F_{0}\right\|_{\varrho_{1}}+\left\|T_{n} F_{1}-F_{1}\right\|_{\varrho_{1}}+\left\|T_{n} F_{2}-F_{2}\right\|_{\varrho_{1}} \geq \frac{r-C \varepsilon}{C}\right\}, \\
D_{0}:=\left\{n \in K:\left\|T_{n} F_{0}-F_{0}\right\|_{\varrho_{1}} \geq \frac{r-C \varepsilon}{3 C}\right\}, \\
D_{1}:=\left\{n \in K:\left\|T_{n} F_{1}-F_{1}\right\|_{\varrho_{1}} \geq \frac{r-C \varepsilon}{3 C}\right\}, \\
D_{2}:=\left\{n \in K:\left\|T_{n} F_{2}-F_{2}\right\|_{\varrho_{1}} \geq \frac{r-C \varepsilon}{3 C}\right\} .
\end{aligned}
$$

Then it is easy to see that $D \subseteq D_{0} \cup D_{1} \cup D_{2}$. Thus (15) yields

$$
\sum_{n \in K: v_{n} \geq r} a_{j n} \leq \sum_{n \in D} a_{j n} \leq \sum_{n \in D_{0}} a_{j n}+\sum_{n \in D_{1}} a_{j n}+\sum_{n \in D_{2}} a_{j n}
$$

from which (7) follows. So by Lemma 2, we have

$$
\text { st }_{A^{-}} \lim _{n}\left\|T_{n} f-f\right\|_{\varrho_{2}}=0 \quad \text { for all } f \in C_{\varrho_{1}} .
$$

Note that if we take $A$ to be the identity matrix $I$, then we immediately get Theorem A.

The next result is a consequence of Theorem 3 .

Corollary 4. Let $\left\{T_{n}\right\}$ be a sequence of positive linear operators from $C_{w}$ into $C_{w}$ for the weight function $w$ defined by $w(x)=1+x^{2}$ and let $A=\left(a_{j n}\right)$ be a non-negative regular summability matrix. Also let $\varrho_{1}$ and $\varrho_{2}$ be weight functions satisfying $(2)$ and consider the sequence $\left\{P_{n}\right\}$ of positive linear operators from $C_{\varrho_{1}}$ into $B_{\varrho_{2}}$ defined by

$$
P_{n}(f(t) ; x)=\frac{\varrho_{1}(x)}{w(x)} T_{n}\left(\frac{1+t^{2}}{\varrho_{1}(t)} f(t) ; x\right) .
$$

If

$$
\operatorname{st}_{A}-\lim _{n}\left\|T_{n} f_{v}-f_{v}\right\|_{w}=0,
$$

where $f_{v}(t)=t^{v}(v=0,1,2)$, then

$$
\text { st }_{A}-\lim _{n}\left\|P_{n} f-f\right\|_{\varrho_{2}}=0 \quad \text { for all } f \in C_{\varrho_{1}} .
$$

Proof. By the definition of the operators $P_{n}$,

$$
P_{n}\left(F_{v} ; x\right)=\frac{\varrho_{1}(x)}{w(x)} T_{n}\left(f_{v} ; x\right) \quad(v=0,1,2),
$$

where $F_{v}(v=0,1,2)$ is as in Theorem 3 . Since, for each $v=0,1,2$,

$$
P_{n}\left(F_{v} ; x\right)-F_{v}(x)=\frac{\varrho_{1}(x)}{w(x)}\left(T_{n}\left(f_{v} ; x\right)-f_{v}(x)\right),
$$


we have

$$
\left\|P_{n} F_{v}-F_{v}\right\|_{\varrho_{1}}=\left\|T_{n} f_{v}-f_{v}\right\|_{w} .
$$

So the assertion follows from Theorem 3.

Let $\varphi$ be a continuous increasing function on $\mathbb{R}$. Now we deal with $A$ statistical approximation in the space $C_{\varrho_{1}}$ with $\varrho_{1}(x)=1+\varphi^{2}(x)$.

LEMMA 5. Let $A=\left(a_{j n}\right)$ be a non-negative regular summability matrix, let $\left\{T_{n}\right\}$ be a sequence of positive linear operators from $C_{\varrho_{1}}$ into $B_{\varrho_{2}}$, and assume that $\varrho_{1}$ and $\varrho_{2}$ satisfy (2). If

$$
\operatorname{st}_{A}-\lim _{n}\left\|T_{n} \varphi^{v}-\varphi^{v}\right\|_{\varrho_{1}}=0 \quad(v=0,1,2),
$$

then

$$
\operatorname{st}_{A}-\lim _{n} \sup _{\|f\|_{\varrho_{1}}=1} \sup _{a \leq x \leq b}\left|T_{n}(f ; x)-f(x)\right|=0
$$

for all $a<b$.

Proof. Let $f \in C_{\varrho_{1}}$. It is shown in [11] that, given $\varepsilon>0$, there exists a number $\delta>0$ such that for all $t \in \mathbb{R}$ and all $x$ satisfying $a \leq x \leq b$ we have

$$
|f(t)-f(x)|<\varepsilon+K_{\varrho_{1}}(x)(\varphi(t)-\varphi(x))^{2},
$$

where

$$
\begin{aligned}
K_{\varrho_{1}}(x) & :=4 M_{f} \varrho_{1}^{2}(x)\left[\frac{1}{\Delta_{\delta}^{2}(\varphi ; x)}+1\right], \\
\Delta_{\delta}(\varphi ; x) & :=\min \{\varphi(x+\delta)-\varphi(x), \varphi(x)-\varphi(x-\delta)\} .
\end{aligned}
$$

Now (17) yields

$$
\begin{aligned}
&\left|T_{n}(f(t) ; x)-f(x)\right| \leq T_{n}(|f(t)-f(x)| ; x)+|f(x)|\left|T_{n}(1 ; x)-1\right| \\
&< \varepsilon T_{n}(1, x)+K_{\varrho_{1}}(x) T_{n}\left((\varphi(t)-\varphi(x))^{2} ; x\right)+|f(x)|\left|T_{n}(1 ; x)-1\right| \\
& \leq(\varepsilon+|f(x)|)\left|T_{n}(1 ; x)-1\right|+\varepsilon+K_{\varrho_{1}}(x) T_{n}\left((\varphi(t)-\varphi(x))^{2} ; x\right) \\
& \leq \varepsilon+(\varepsilon+|f(x)|)\left|T_{n}(1 ; x)-1\right| \\
&+K_{\varrho_{1}}(x)\left\{\left|T_{n}\left(\varphi^{2}(t) ; x\right)-\varphi^{2}(x)\right|+2|\varphi(x)|\left|T_{n}(\varphi(t) ; x)-\varphi(x)\right|\right. \\
&\left.+\varphi^{2}(x)\left|T_{n}(1 ; x)-1\right|\right\} \\
&=+\left\{\varepsilon+|f(x)|+K_{\varrho_{1}}(x)+\varphi^{2}(x)\right\}\left|T_{n}(1 ; x)-1\right| \\
&+2 K_{\varrho_{1}}(x)|\varphi(x)|\left|T_{n}(\varphi(t) ; x)-\varphi(x)\right|+K_{\varrho_{1}}(x)\left|T_{n}\left(\varphi^{2}(t) ; x\right)-\varphi^{2}(x)\right| .
\end{aligned}
$$

So we get

$$
\begin{aligned}
u_{n} & :=\sup _{\|f\|_{\varrho_{1}}=1} \sup _{a \leq x \leq b}\left|T_{n}(f(t) ; x)-f(x)\right| \\
& \leq \varepsilon+C\left\{\left\|T_{n} 1-1\right\|_{\varrho_{1}}+\left\|T_{n} \varphi-\varphi\right\|_{\varrho_{1}}+\left\|T_{n} \varphi^{2}-\varphi^{2}\right\|_{\varrho_{1}}\right\}
\end{aligned}
$$


where

$$
\begin{aligned}
C:=\max \left\{\operatorname { s u p } _ { a \leq x \leq b } \varrho _ { 1 } ( x ) \left(\varepsilon+|f(x)|+K_{\varrho_{1}}(x)\right.\right. & \left.+\varphi^{2}(x)\right), \\
& \left.\sup _{a \leq x \leq b} 2 \varrho_{1}(x) K_{\varrho_{1}}(x)|\varphi(x)|\right\} .
\end{aligned}
$$

Now for a given $r>0$, choose $\varepsilon>0$ such that $\varepsilon<r$. Define

$$
\begin{aligned}
D:=\left\{n:\left\|T_{n} 1-1\right\|_{\varrho_{1}}+\left\|T_{n} \varphi-\varphi\right\|_{\varrho_{1}}+\left\|T_{n} \varphi^{2}-\varphi^{2}\right\|_{\varrho_{1}} \geq \frac{r-\varepsilon}{C}\right\}, \\
D_{0}:=\left\{n:\left\|T_{n} 1-1\right\|_{\varrho_{1}} \geq \frac{r-\varepsilon}{3 C}\right\}, \\
D_{1}:=\left\{n:\left\|T_{n} \varphi-\varphi\right\|_{\varrho_{1}} \geq \frac{r-\varepsilon}{3 C}\right\}, \\
D_{2}:=\left\{n:\left\|T_{n} \varphi^{2}-\varphi^{2}\right\|_{\varrho_{1}} \geq \frac{r-\varepsilon}{3 C}\right\} .
\end{aligned}
$$

Then it is easy to see that $D \subseteq D_{0} \cup D_{1} \cup D_{2}$. By (18) we have

$$
\sum_{n: u_{n} \geq r} a_{j n} \leq \sum_{n \in D} a_{j n} \leq \sum_{n \in D_{0}} a_{j n}+\sum_{n \in D_{1}} a_{j n}+\sum_{n \in D_{2}} a_{j n} .
$$

Letting $n \rightarrow \infty$ in (19) and using (16) we conclude that

$$
\operatorname{st}_{A^{-}} \lim _{n} \sup _{\|f\|_{\varrho_{1}=1}} \sup _{a \leq x \leq b}\left|T_{n}(f ; x)-f(x)\right|=0,
$$

which completes the proof.

Assume now that $\varrho_{1}:=1+\varphi^{2}$ and $\varrho_{2}$ satisfy (2). Then by Lemmas 2 and 5 we get the following $A$-statistical Korovkin type approximation theorem.

TheOREM 6. Let $A=\left(a_{j n}\right)$ and $\left\{T_{n}\right\}$ be as in Lemma 5. Then (8) holds if and only if $\left\{T_{n}\right\}$ satisfies (16).

Proof. Since $\varphi^{v} \in C_{\varrho_{1}}(v=0,1,2),(8)$ implies (16). Assume now that $\left\{T_{n}\right\}$ satisfies (16). By Lemma 5 we have

$$
\mathrm{st}_{A^{-}} \lim _{n} \sup _{\|f\|_{\varrho_{1}}=1} \sup _{-s \leq x \leq s}\left|T_{n}(f ; x)-f(x)\right|=0
$$

for any $s \in \mathbb{R}$. Also, as in the proof of Theorem 3 we can find a positive number $M$ such that $\delta_{A}\left\{n \in \mathbb{N}:\left\|T_{n}\right\|_{C_{\varrho_{1}} \rightarrow B_{\varrho_{1}}} \leq M\right\}=1$. It follows from Lemma 2 that

$$
\operatorname{st}_{A^{-}} \lim _{n}\left\|T_{n} f-f\right\|_{\varrho_{2}}=0 \quad \text { for all } f \in C_{\varrho_{1}} \text {. }
$$

3. Concluding remarks. In this section we deal with an example of a sequence of positive linear operators to which Theorem A does not apply but our Theorem 3 does. 
EXAMPLE. Let $\varrho_{1}$ and $\varrho_{2}$ be weight functions satisfying (2) and let $\left\{L_{n}\right\}$ be a sequence of positive linear operators from $C_{\varrho_{1}}$ into $B_{\varrho_{2}}$ satisfying one of the two equivalent properties stated in Theorem A. Assume that $A=\left(a_{n k}\right)$ is a non-negative regular summability matrix such that $\lim _{j} \max _{n}\left\{a_{j n}\right\}=0$; then $A$-statistical convergence is stronger than convergence. So there is a sequence $\left(u_{n}\right)$ which is $A$-statistically null but not convergent [17]. Without loss of generality we may assume that $\left(u_{n}\right)$ is non-negative. Now define the sequence $\left\{T_{n}\right\}$ of positive linear operators mapping $C_{\varrho_{1}}$ into $B_{\varrho_{2}}$ by $T_{n}(f)=\left(1+u_{n}\right) L_{n}(f)$ for $f \in C_{\varrho_{1}}$. Observe that $\left\{u_{n} L_{n}(f)\right\}$ does not tend to zero because $L_{n}(f) \rightarrow f$ for all $f \in C_{\varrho_{1}}$ and $\left(u_{n}\right)$ is divergent. Hence the sequence $\left\{\left\|T_{n} f-f\right\|_{\varrho_{2}}\right\}$ does not tend to zero either, but it is an $A$-statistically null sequence for all $f \in C_{\varrho_{1}}$.

Acknowledgements. The authors wish to thank the referee for his/her valuable suggestions which improved the paper considerably.

\section{References}

[1] F. Altomare and M. Campiti, Korovkin-Type Approximation Theory and its Applications, de Gruyter Stud. Math. 17, de Gruyter, Berlin, 1994.

[2] R. Bojanić and F. Cheng, Estimates for the rate of approximation of functions of bounded variation by Hermite-Fejér polynomials, in: CMS Conf. Proc. 3, 1983, 5-17.

[3] R. Bojanić and M. K. Khan, Summability of Hermite-Fejér interpolation for functions of bounded variation, J. Natur. Sci. Math. 32 (1992), 5-10.

[4] J. S. Connor, On strong matrix summability with respect to a modulus and statistical convergence, Canad. Math. Bull. 32 (1989), 194-198.

[5] R. A. Devore, The Approximation of Continuous Functions by Positive Linear Operators, Lecture Notes in Math. 293, Springer, Berlin, 1972.

[6] O. Duman and C. Orhan, Statistical approximation in the space of locally integrable functions, Publ. Math. Debrecen 63 (2003), 133-134.

[7] H. Fast, Sur la convergence statistique, Colloq. Math. 2 (1951), 241-244.

[8] A. R. Freedman and J. J. Sember, Densities and summability, Pacific J. Math. 95 (1981), 293-305.

[9] J. A. Fridy, On statistical convergence, Analysis 5 (1985), 301-313.

[10] J. A. Fridy and C. Orhan, Statistical limit superior and limit inferior, Proc. Amer. Math. Soc. 125 (1997), 3625-3631.

[11] A. D. Gadjiev, On P. P. Korovkin type theorems, Mat. Zametki 20 (1976), 781-786 (in Russian).

[12] - The convergence problem for a sequence of positive linear operators on unbounded sets, and theorems analogous to that of P. P. Korovkin, Soviet Math. Dokl. 15 (1974), 1433-1436.

[13] A. D. Gadjiev and C. Orhan, Some approximation theorems via statistical convergence, Rocky Mountain J. Math. 32 (2002), 129-138.

[14] G. H. Hardy, Divergent Series, Oxford Univ. Press, London, 1949.

[15] E. Kolk, Statistically convergent sequences in normed spaces, in: Reports of Conference, "Methods of Algebra and Analysis", Tartu, 1988, 63-66. 
[16] E. Kolk, The statistical convergence in Banach spaces, Acta Comment. Tartuensis 928 (1991), 41-52.

[17] —, Matrix summability of statistically convergent sequences, Analysis 13 (1993), 77-83.

[18] P. P. Korovkin, Linear Operators and Theory of Approximation, Hindustan Publ. Co., Delhi, 1960.

[19] H. I. Miller, A measure theoretical subsequence characterization of statistical convergence, Trans. Amer. Math. Soc. 347 (1995), 1811-1819.

Department of Mathematics

Faculty of Science

Ankara University

Tandogan 06100, Ankara, Turkey

E-mail: oduman@science.ankara.edu.tr

orhan@science.ankara.edu.tr

Received March 6, 2003

Revised version July 1, 2003 